\title{
Fourier analysis versus multiple linear regression to analyse pressure-flow data during artificial ventilation
}

\author{
R. Peslin, C. Gallina, C. Saunier, C. Duvivier
}

Fourier analysis versus multiple linear regression to analyse pressure-flow data during artificial ventilation. R. Peslin, C. Gallina, C. Saunier, C. Duvivier. ERS Journals Ltd 1994.

ABSTRACT: Respiratory resistance (Rrs) and elastance (Ers) are commonly measured in artificially-ventilated patients or animals by multiple linear regression of airway opening pressure (Pao) versus flow $\left(V^{\prime}\right)$ and volume $\left(V^{\prime}\right)$, according to the first order model: $\mathbf{P a o}=\mathbf{P}_{0}+\mathbf{E r s} \cdot \mathrm{V}+\mathrm{Rrs} \cdot \mathrm{V}^{\prime}$, where $\mathbf{P}_{0}$ is the static recoil pressure at end-expiration. An alternative way to obtain Rrs and Ers is to derive them from the Fourier coefficients of Pao and $V^{\prime}$ at the breathing frequency. A potential advantage of the second approach over the first is that it should be insensitive to a zero offset on ' $V^{\prime}$ and to the corresponding volume drift.

The two methods were assessed comparatively in six tracheotomized, paralysed and artificially ventilated rabbits with and without adding to $V^{\prime}$ an offset equal to $5 \%$ of the mean unsigned flow.

The 5\% flow offset did not modify the results of Fourier analysis, but increased Rrs and Ers from linear regression by $15.8 \pm 4.6 \%$ and $4.55 \pm 0.64 \%$, respectively.

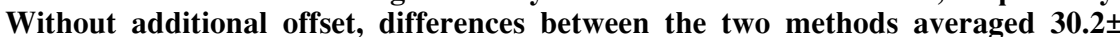
$14.0 \%$ for Rrs and $9.3 \pm 6.2 \%$ for Ers. The differences almost completely disappeared $(2.47$ and $0.61 \%$, respectively) when the flow signal was zero-corrected using the assumption that inspired and expired volumes were the same. After induced bronchoconstriction, however, Ers was still slightly larger by linear regression than by Fourier analysis, which may result from nonlinearities and/or frequency dependence of the parameters.

We conclude that the regression method requires zero flow correction and that Fourier analysis is an attractive alternative.

Eur Respir J., 1994, 7, 2241-2245.
Unité 14 de Physiopathologie Respiratoire, Institut National de la Santé et de la Recherche Médicale, Université de Nancy 1, Vandoeuvre-les-Nancy, France.

Correspondence: R. Peslin

Unité 14 INSERM

Physiopathologie Respiratoire C.O. No. 10

54511 Vandoeuvre-les-Nancy cedex France

Keywords: Data analysis monitoring

respiratory mechanics

Received: April 191994

Accepted after revision August 11994
Total or lung respiratory resistance (Rrs) and elastance (Ers) are commonly measured in artificially ventilated humans [1-5] or animals [6, 7] by analysing the relationship between airway opening pressure (Pao) and flow $\left(V^{\prime}\right)$ with the first order model:

$$
\mathrm{PaO}=\mathrm{P}_{0}+\mathrm{Ers} \cdot \mathrm{V}+\mathrm{Rrs} \cdot \mathrm{V}^{\prime}
$$

where $\mathrm{V}$ is lung volume, as obtained by electrical or numerical integration of the flow signal, and $\mathrm{P}_{0}$ is the static recoil pressure at the lung volume at which the integration is started (in this study the end-expiratory volume). As initially proposed by WALD et al. [8] and UHL and LEWIS [9] for obtaining lung elastance and resistance, Ers, Rrs and $\mathrm{P}_{0}$ are usually computed by multiple linear regression (MLR) of Pao versus $\mathrm{V}$ and $\mathrm{V}$ '. A potential problem with the method is that a zero offset of the flow signal, and the corresponding volume drift, are likely to be responsible for errors on the coefficients. An alternative method, theoretically immune from that source of error, is to compute the Fourier coefficients [10] of Pao and $\mathrm{V}^{\prime}$ at the breathing frequency $(f)$ and, from them, the real (Re) and imaginary (Im) parts of total respiratory impedance at that frequency. Then, according to the same model:
Rrs $=\operatorname{Re}$
Ers $=-2 \cdot \pi \cdot f \cdot \operatorname{Im}$

The aims of this investigation were: 1) to quantify the influence of a flow offset on the coefficients obtained with the MLR method; 2) to compare the MLR method and Fourier analysis (FA); and 3) to assess the value of a simple algorithm for zero flow correction.

\section{Methods}

The study was performed in six New Zealand rabbits with a body weight of $2.0-2.7 \mathrm{~kg}$. The animals were anaesthetized with sodium thiopental $\left(15-20 \mathrm{mg} \cdot \mathrm{kg}^{-1}\right.$ i.v. and additional doses as needed during the study) and tracheotomized. They were artificially ventilated by a piston-pump ventilator (Harvard Rodent ventilator, type 683 ), with a tidal volume of $10 \mathrm{ml} \cdot \mathrm{kg}^{-1}$ and a frequency of 50 strokes $\cdot \mathrm{min}^{-1}$. They were paralysed with vecuronium bromide $(0.8 \mathrm{mg}$, followed by continuous infusion of $\left.0.4 \mathrm{mg} \cdot \mathrm{h}^{-1}\right)$. 
$\mathrm{V}^{\prime}$ was measured with a heated Fleisch No. 00 pneumotachograph interposed between the tracheal cannula and the ventilator, and connected to a differential pressure transducer (Honeywell type 176/14 PC). The zero of the flow channel was carefully set at the beginning of the experiment, but was not readjusted afterward. Pao was sampled close to the cannula and measured with a similar transducer. The responses of the two transducers were matched within $2 \%$ of amplitude and $2^{\circ}$ of phase up to $30 \mathrm{~Hz}$. After analogue low-pass filtering at $35 \mathrm{~Hz}$, the two signals were digitized for periods of $10 \mathrm{~s}$ with a sampling rate of $100 \mathrm{~Hz}$, and processed by a personal computer equipped with a 12 bits analogue-digital conversion board (Digimétrie PCLab). The measurements were repeated at 1 minute intervals before and during induced bronchoconstriction. The latter was obtained by connecting the air inlet of the ventilator to a nebulizer (Intersurgical, 1493-000 Gould RD, Twickenham, Middlesex, UK) delivering histamine $176 \mu \mathrm{g} \cdot l^{-1}$ of gas, with a flow in excess of that drawn by the ventilator. The aerosol lasted for $5 \mathrm{~min}$ and the total amount of histamine delivered to the animal was $1,100 \mu \mathrm{g}$.

The first step in the data processing was to identify the beginning and end of the successive respiratory cycles. The MLR method was then applied on a cycleper-cycle basis and the data from the 7-8 recorded cycles were averaged. The FA method was also applied on a cycle-per-cycle basis by computing the Fourier coefficients $(\mathrm{a}, \mathrm{b})$ of the pressure and flow signals according to:

$$
a_{x}=\sum_{i=1}^{n} x_{i} \cdot \cos (i \cdot d \varphi) \text { and } b_{x}=\sum_{i=1}^{n} x_{i} \cdot \sin (i \cdot d \varphi)
$$

where $\mathrm{x}$ stands for Pao or $\mathrm{V}^{\prime}, \mathrm{n}$ is the number of data points in the cycle and $d \varphi=2 \pi / n$. No windowing was used, since the length of the data block was equal to that of the cycle.

The data were analysed both by MLR and FA in four different ways: 1) using the flow data as they were recorded; 2) after adding numerically to the flow values a quantity equal to $5 \%$ of the mean rectified flow (inspiratory offset); 3) after subtracting a similar quantity (expiratory offset); and 4) after drift correction of the flow signal. The latter was estimated from the change in end-expiratory lung volume between the first and the last recorded cycle; that change was assumed to be entirely due to a flow offset, and its value (change in volume per time unit) was subtracted from the data. The values of the coefficients obtained from 10 consecutive measurements at $1 \mathrm{~min}$ intervals were averaged. After histamine, the selected period was that when bronchoconstriction was maximal, as judged from Rrs.

Statistical analysis included t-test for paired data and small samples, and simple linear regression.

\section{Results}

As expected, the coefficients obtained by FA were totally unchanged when adding a positive or a negative offset to the flow values. In contrast, systematic changes were seen with the MLR method, as illustrated in figure 1 for the data obtained before histamine: a small inspiratory flow offset systematically and significantly increased both Ers $(4.55 \pm 0.64 \% ; \mathrm{p}<0.001)$ and Rrs $(15.8 \pm$ $4.6 \%, \mathrm{p}<0.001)$; it also decreased $\mathrm{P}_{0}$ which fell from $0.18 \pm 0.10$ to $0.11 \pm 0.11 \mathrm{kPa}(\mathrm{p}<0.01)$. Symmetrical changes were seen with an expiratory flow offset.

The coefficients obtained with the two methods, using the original flow data, are compared in figure $2 \mathrm{a}$. Ers and Rrs were systematically lower by MLR than by FA $(\mathrm{p}<0.001)$, with differences averaging $-9.3 \pm 6.2 \%$ for Ers and $-30.2 \pm 14.0 \%$ for Rrs. Analysing the same data after correcting the flow values for the estimated
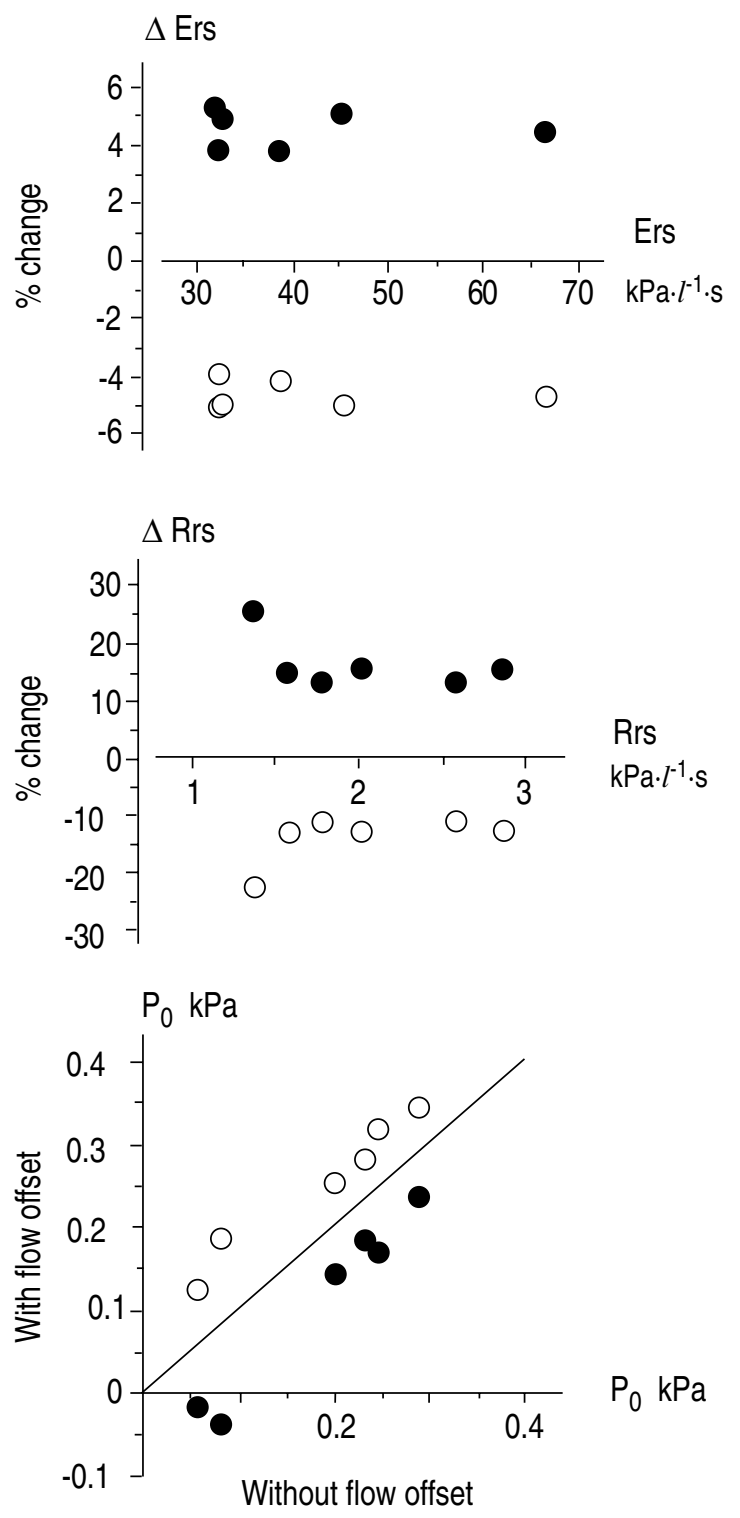

Fig 1. - Influence of a small flow offset (5\% of mean unsigned flow) on the values of total respiratory elastance (Ers) and resistance (Rrs), and of end-expiratory recoil pressure $\left(\mathrm{P}_{0}\right)$ obtained by multiple linear regression in unchallenged rabbits. For Ers and Rrs the changes are shown as a percentage of the value obtained with the original flow data. - flow offset in inspiratory direction; $\bigcirc$ : flow offset in expiratory direction. Identity line is shown in bottom panel. 
a)

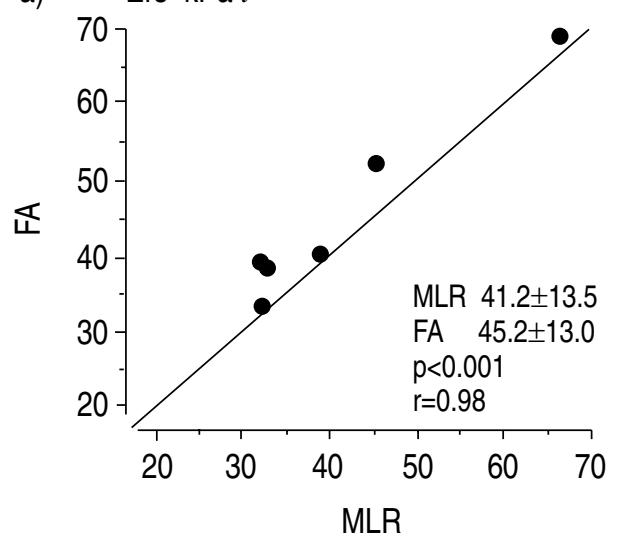

b) $\quad$ Ers $\mathrm{kPa} \cdot \mathrm{l}^{-1}$

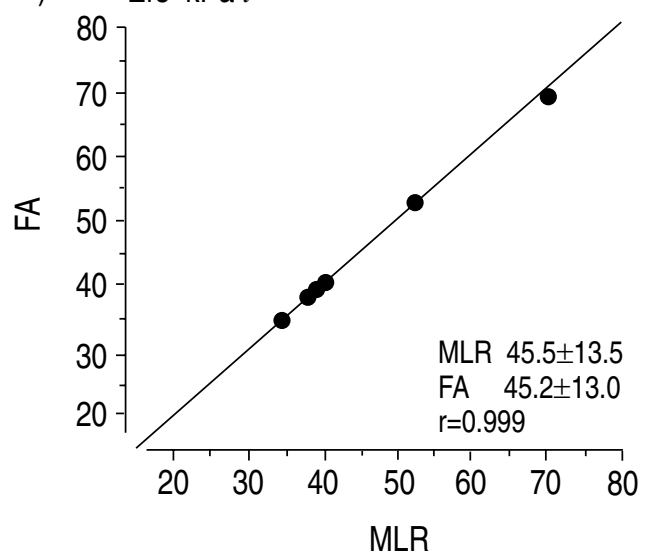

Rrs $\mathrm{kPa} \cdot \mathrm{l}^{-1} \cdot \mathrm{s}$

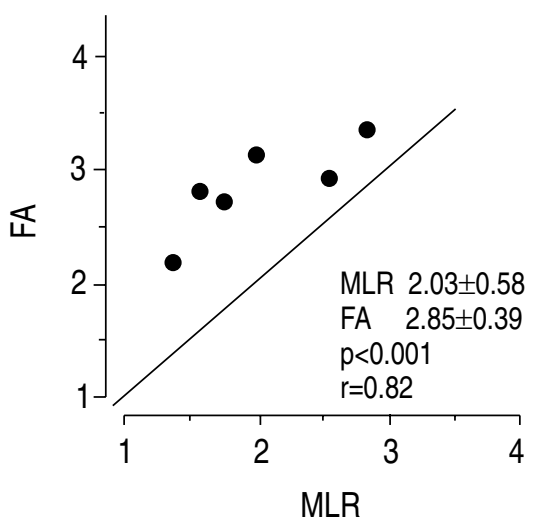

$\operatorname{Rrs~} \mathrm{kPa} \cdot l^{-1} \cdot \mathrm{s}$

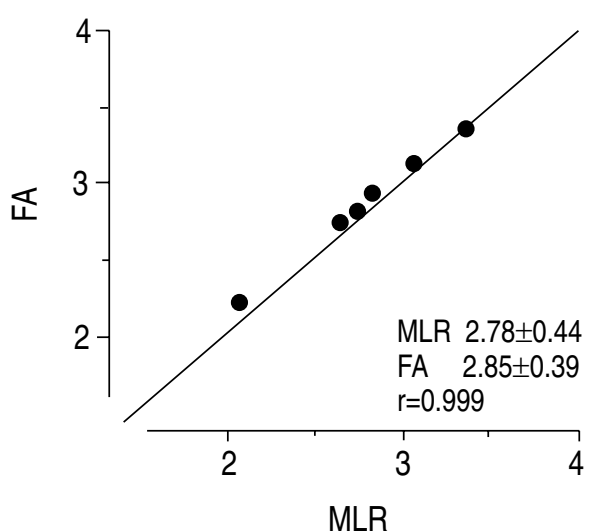

Fig 2. - Comparison of the coefficients obtained by multiple linear regression (MLR) and by Fourier analysis (FA). a) with the original flow data; b) after correcting the flow data for the estimated drift. For further abbreviations see legend to figure 1 . Values are mean \pm SD; identity lines are shown.
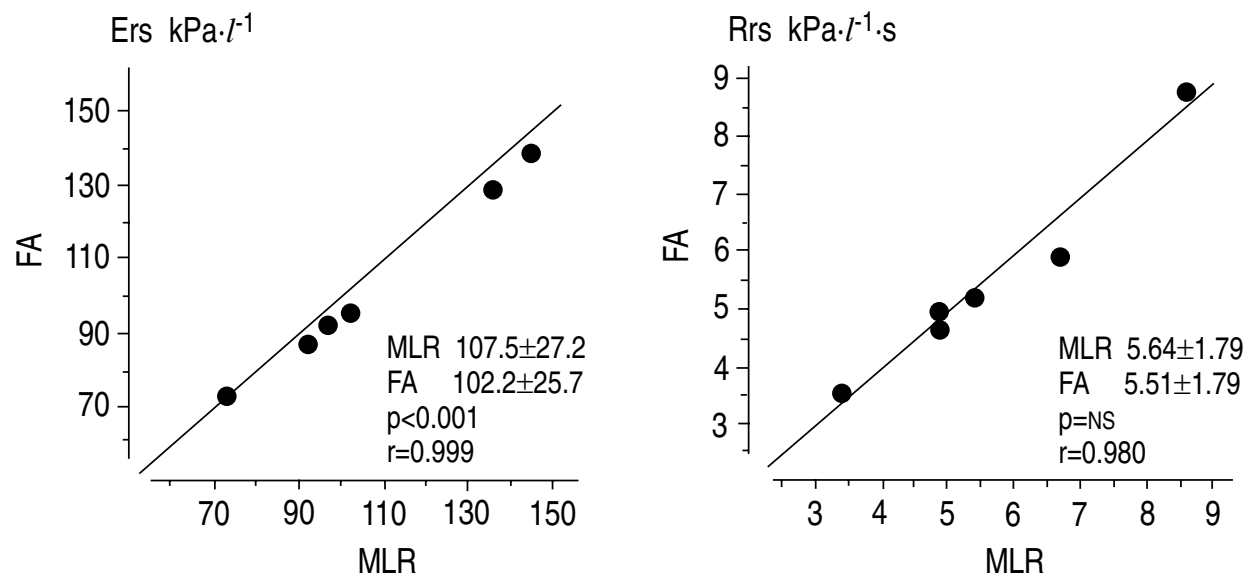

Fig 3. - Comparison of the coefficients obtained by multiple linear regression (MLR) and by Fourier analysis (FA) from the measurements made during acute bronchoconstriction. The flow data were corrected for the estimated drift. For further abbreviations see legend to figure 1 . Values are mean \pm sD; identity lines are shown.

drift (fig. 2b) showed that the correction considerably improved the agreement between the two methods: the differences were not significant and averaged only $0.61 \pm$ $0.93 \%$ for Ers; they were a little larger $(-2.5 \pm 2.2 \%)$ and still slightly significant $(\mathrm{p}<0.05)$ for Rrs. The correlations between the coefficients obtained with the two methods were also much improved (fig. 2). As shown in fig. 2, zero-flow correction increased both MLR
Ers $(\mathrm{p}<0.02)$ and Rrs $(\mathrm{p}<0.02)$; it decreased $\mathrm{P}_{0}(0.011 \pm$ $0.084 \mathrm{kPa}$ compared to $0.184 \pm 0.095 \mathrm{kPa}$; $\mathrm{p}<0.01$ ).

Figure 3 compares the coefficients obtained during induced bronchoconstriction, when the flow data were drift-corrected. For Ers, the agreement was still satisfactory ( $\mathrm{r}=0.999)$ but the values obtained by MLR were $5.17 \pm 1.52 \%$ larger than by FA $(\mathrm{p}<0.001)$. For Rrs, the differences were smaller $(2.2 \pm 6.3 \%)$ and not significant. 


\section{Discussion}

This study first demonstrated that a slight change in the flow baseline could substantially modify the values of Rrs and Ers as obtained by MLR. One may point out that the flow offset used to obtain the data in figure 1 was far from unrealistic. Indeed, transposed to humans, $5 \%$ of the mean unsigned flow corresponds to only 10 $\mathrm{ml} \cdot \mathrm{s}^{-1}$ for a ventilation of $6 \mathrm{l} \cdot \mathrm{min}^{-1}$. This is corroborated by the data in figure $2 \mathrm{a}$ which showed that, although the zero of the flow channel was adjusted at the beginning of the experiment, systematic differences were seen between MLR and FA, suggestive of a small drift of the flow signal in the expiratory direction.

We monitored the stability of the transducers and electronics used in this study, and observed a drift amounting to about $4 \mathrm{~Pa}$ over a period of $2 \mathrm{~h}$, which may well account for the results. Temperature and water vapour pressure differences between inspired and expired gas, as well as the difference between $\mathrm{O}_{2}$ uptake and $\mathrm{CO}_{2}$ output, should also have contributed to the overall volume drift. On the other hand, the data in figure $2 \mathrm{~b}$ suggest that a good estimate of the flow offset can be obtained using the assumption that the end-expiratory volume is the same at the beginning and at the end of the sampling period. Of course, such an assumption cannot be made if the ventilatory condition is not perfectly steady. Also, the zero error may be misestimated if there is a leak in the respiratory circuit, responsible for true differences between the inspired and expired volumes across the pneumotachograph.

Whilst zero correction of the flow signal considerably improved the agreement between MLR and FA, some differences persisted, particularly during acute bronchoconstriction. This does not necessarily reflect a misestimation of the flow offset. Indeed, the two approaches may be expected to provide different results in two situations: when respiratory mechanical properties are frequency dependent and when the system is nonlinear. The differences will arise from the fact that FA gives the relationship between pressure and flow at a specific frequency, whilst MLR uses all the available information; they are expected to depend upon the flow waveform. Using computer simulation with a flow waveform close to that delivered during artificial ventilation (constant inspiratory flow, passive expiration), we tried to estimate the possible magnitude of such differences in three instances: viscoelastic behaviour, flow dependence of resistance, volume dependence of elastance.

Lung and chest wall viscoelasticity may be responsible for a negative frequency dependence of Rrs and a positive frequency dependence of Ers [11-13]; this was modelled by a pure elastance $\left(\mathrm{E}_{0}\right)$ in parallel with a newtonian resistance $\left(\mathrm{R}_{0}\right)$, and with a Maxwell element $\left(\mathrm{E}_{1}, \mathrm{R}_{1}\right)[14]$ :

$$
\mathrm{P}+\tau \cdot \mathrm{P}^{\prime}=\mathrm{E}_{0} \cdot \mathrm{V}+\left(\mathrm{R}_{0}+\mathrm{R}_{1}+\mathrm{E}_{0} \cdot \tau\right) \cdot \mathrm{V}^{\prime}+\mathrm{R}_{0} \cdot \tau \cdot \mathrm{V}^{\prime \prime}
$$

where $\tau$ is the time constant of the viscoelastic element $\left(\mathrm{R}_{0} / \mathrm{E}_{0}\right)$, and $\mathrm{P}^{\prime}$ and $\mathrm{V}^{\prime \prime}$ are the time derivatives of $\mathrm{P}$ and $\mathrm{V}^{\prime}$, respectively; the model may also account for mechanical inhomogeneity [15]. Using various sets of coefficients, such that the frequency dependence of effective resistance and elastance was large in the vicinity of the breathing frequency, showed that almost identical values of Ers were obtained by MLR and by FA, but that FA provided slightly larger values of Rrs. An example is shown in table 1.

A nonlinearity of the pressure-flow relationship was modelled according to Rohrer's equation [16]. Simulation showed that it resulted in slightly larger Rrs and lower Ers by FA than by MLR. However, the effect was minimal, even when the nonlinear term was responsible for a large part of the resistance (table 1).

Finally, a nonlinearity of the pressure-volume curve was modelled by assuming that elastance increased linearly with lung volume $\left(\mathrm{Ers}=\mathrm{E}_{0}+\mathrm{Kv} \cdot \mathrm{V}\right)[3,4]$. In that situation, Rrs and Ers were systematically larger by MLR than by FA. The differences were of the order of $2 \%$ when half of the elastance was due to the nonlinear term (table 1).

These computer simulations show that, whatever the situation, the differences between MLR and FA due to nonlinearites or frequency dependence of the coefficients are unlikely to be very large. However, they may explain some of the differences seen in this study after drift-correction. In particular, the slightly larger values of Ers obtained by MLR during acute bronchoconstriction could reflect a volume dependence of elastance, or a combination of the above situations.

The errors seen with the MLR method when a flow offset is present originate both from the drift of the

Table 1. - Influence of frequency dependence and of nonlinearity on coefficients obtained by MLR and by FA (computer simulation)

\begin{tabular}{ccccc}
\hline Model & MLR & Ers & RA & MLR \\
\hline $\mathrm{P}+1 \mathrm{P}^{\prime}=20 \mathrm{~V}+45 \mathrm{~V}^{\prime}+2.5 \mathrm{~V}^{\prime \prime}$ & 39.05 & 39.01 & 5.70 & 5.89 \\
$\mathrm{P}=20 \mathrm{~V}+\left(5+58 \mathrm{IV}^{\prime} \mathrm{l}\right) \cdot \mathrm{V}^{\prime}$ & 18.91 & 18.79 & 10.14 & 10.21 \\
$\mathrm{P}=(20+400 \mathrm{~V}) \cdot \mathrm{V}+5 \mathrm{~V}^{\prime}$ & 41.13 & 40.15 & 5.00 & 4.93
\end{tabular}

From top to bottom, viscoelastic model, flow dependence of resistance, volume dependence of elastance. In all instances: inspiratory flow $0.1 l \cdot \mathrm{s}^{-1}$, inspiratory time $0.5 \mathrm{~s}$, end-inspiratory pause $0.1 \mathrm{~s}$, passive expiration with expiratory time $0.6 \mathrm{~s}$. Units for plausible values in rabbits: $\mathrm{P}$ in $\mathrm{kPa}, \mathrm{V}$ in $\mathrm{l}$, time in $\mathrm{s}$. See text for further explanation. MLR: multiple linear regression; FA: Fourier analysis; Ers: total respiratory elastance; Rrs: total respiratory resistance; P: pressure; V: volume; V': flow; V", P': time derivatives of $\mathrm{V}^{\prime}$ and $\mathrm{P}$. 
volume data obtained by integration of the flow signal, and from the inaccuracy of the flow itself. If the volume-related error were to dominate the picture, an interesting approach would be to fit the data to a differentiated version of Equation (1), which eliminates the need to compute $\mathrm{V}$ :

$$
\mathrm{Pao}^{\prime}=\mathrm{Ers} \cdot \mathrm{V}^{\prime}+\mathrm{Rrs} \cdot \mathrm{V}^{\prime \prime}
$$

where Pao' is the time derivatives of Pao. We tried that variant on our data using equation 5 in the form: $\mathrm{Pao}^{\prime} / \mathrm{V}^{\prime \prime}=$ $\mathrm{Rrs}+\mathrm{Ers} \cdot \mathrm{V}^{\prime} / \mathrm{V}^{\prime \prime}$. In addition to an increased intrasubject variability of the results, we observed systematically and significantly lower values of Rrs when the flow offset was corrected for than when it was not (on average $1.99 \pm 0.77$ vs $\left.2.49 \pm 1.00 \mathrm{kPa} \cdot \mathrm{s} \cdot l^{-1}\right)$. The difference on Ers was much lower and not significant. This suggests that the flow offset may still be responsible for substantial errors, even when the volume drift is not an issue.

Whilst the FA may be quite valuable when the flow offset cannot be corrected for, an obvious drawback is that it does not provide the coefficient $\mathrm{P}_{0}$, which is of practical interest to detect intrinsic positive end-expiratory pressure (PEEP) in artificially-ventilated humans [2, 3] or animals. This may be remedied as follows. Introducing the value of the flow offset (Vo'), equation (1) may be rewritten in the discrete form:

$$
\mathrm{Pao}_{\mathrm{i}}=\mathrm{P}_{0}+\operatorname{Rrs} \cdot\left(\mathrm{V}_{\mathrm{i}}^{\prime}-\mathrm{Vo}^{\prime}\right)+\operatorname{Ers}\left(\mathrm{V}_{\mathrm{i}}-\mathrm{i} \cdot \mathrm{Vo}^{\prime} \cdot \mathrm{dt}\right)
$$

where $\mathrm{dt}$ is the reciprocal of the sampling frequency. $\mathrm{Vo}^{\prime} \cdot \mathrm{dt}$ represents the error on the volume estimate which is made at each step of the numerical integration. Then:

$\mathrm{PaO}_{\mathrm{i}}-\mathrm{Rrs} \cdot \mathrm{V}_{\mathrm{i}}^{\prime}-\mathrm{Ers} \cdot \mathrm{V}_{\mathrm{i}}=\mathrm{P}_{0}-\mathrm{Rrs} \cdot \mathrm{Vo}^{\prime}-\mathrm{Vo}^{\prime} \cdot \mathrm{dt} \cdot$ Ers $\cdot \mathrm{i}$ (7)

which is of the form $y=a+b \cdot x$ with $a=P_{0}-R r s \cdot V o$ and $\mathrm{b}=-\mathrm{Vo}^{\prime} \cdot \mathrm{dt} \cdot$ Ers. Using the values of Rrs and Ers obtained by FA, it is therefore possible to obtain $\mathrm{P}_{0}$ and, additionally, $\mathrm{Vo}^{\prime}$ by simple linear regression of the left member versus the index i. Applying that approach to our data provided values of $\mathrm{P}_{0}$ which were extremely similar to those obtained by MLR after zero-correction of the flow signal: the two estimates were highly correlated $(r=0.999)$, were not significantly different, and their unsigned differences averaged only $0.0035 \pm 0.0043 \mathrm{kPa}$.

In this study, we only applied FA to the fundamental frequency of the breathing signals. Then, the analysis provided only two coefficients which were interpreted in terms of Rrs and Ers on the basis of a first order model. If the model were more complex, for instance including airway inertance [17], or accounting for viscoelasticity [14] or mechanical inhomogeneity [15], a multiple frequency approach would be necessary to estimate all the parameters. Whilst the MLR method can, in principle, be applied to higher order models, it also requires that the signals contain harmonics of the fundamental frequency with sufficient energy.

We conclude that: 1) the multiple linear regression method requires zero flow correction; 2) in the absence of leaks, that correction may easily be achieved provided lung volume is sufficiently steady; 3 ) Fourier analysis is an attractive alternative, which does not require zero flow correction.
Acknowledgements: The authors are grateful to B. Clement for editing the manuscript and to M.C. Rohrer for the illustrations.

\section{References}

1. Benhamou D, Lorino AM, Lorino H, Zerah F, Harf A. Automated measurement of respiratory mechanics in anaesthetized ventilated patients. Bull Eur Physiopathol Respir 1987; 23: 423-428.

2. Nicolai T, Lanteri C, Freezer N, Sly PD. Noninvasive determination of alveolar pressure during mechanical ventilation. Eur Respir J 1991; 4: 1275-1283.

3. Peslin R, Felicio Da Silva J, Chabot F, Duvivier C. Respiratory mechanics studied by multiple linear regression in unsedated ventilated patients. Eur Respir J 1992; 5: 871-878.

4. Rousselot JM, Peslin R, Duvivier C. Evaluation of the multiple linear regression method to monitor respiratory mechanics in ventilated neonates and young children. Pediatr Pulmonol 1992; 13: 161-168.

5. Wensley DF, Noonan P, Seear MD, Werner H, Pirie GE. Pilot study for the development of a monitoring device for ventilated children. Pediatr Pulmonol 1991; 11: 272-279.

6. Bates JHT, Shardonofsky F, Stewart DE. The lowfrequency dependence of respiratory system resistance and elastance in normal dogs. Respir Physiol 1989; 78: 369-382.

7. Dewachter P, Saunier CG, Duvivier C, Peslin R, Laxenaire C. Changes in inspired gas composition and experimental bronchospasm in the rabbit. Respir Physiol 1992; 90: 261-269.

8. Wald A, Jason D, Murphy TW, et al. A computer system for respiratory parameters. Comput Biomed Res 1969; 2: 411-429.

9. Uhl RR, Lewis FJ. Digital computer calculation of human pulmonary mechanics using a least squares fit technique. Comput Biomed Res 1974; 7: 489-495.

10. Feyman RP, Leighton RB, Sands M. The Feynman Lectures on Physics. Vol I, Mechanics part 2. Bilingual edn. London Addison-Wesley Publ. Co. 1969; Chap. 50, pp. 1-14.

11. Hildebrandt, J. Pressure-volume data of cat lung interpreted by a plastoelastic, linear viscoelastic model. $J$ Appl Physiol 1970; 28: 365-372.

12. Hantos Z, Daroczy B, Suki B, Galgoczy G, Csendes T. Forced oscillatory impedance of the respiratory system at low frequencies. J Appl Physiol 1986; 60: 123-132.

13. Barnas GM, Yoshino K, Loring SH, Mead J. Impedance and relative displacements of the relaxed chest wall up to $4 \mathrm{~Hz}$. J Appl Physiol 1987; 62: 71-81.

14. Similowski T, Bates JHT. Two-compartment modelling of respiratory system mechanics at low frequencies: gas redistribution or tissue rheology. Eur Respir J 1991; 4: $353-358$.

15. Otis $\mathrm{AB}$, McKerrow $\mathrm{CB}$, Bartlett RA, et al. Mechanical factors in distribution of pulmonary ventilation. $J$ Appl Physiol 1956; 8: 427-443.

16. Rohrer R. Der Strömungswiderstand in den menschlichen Atemwegen und der Einfluss der unregelmassigen Verzweigung des bronchial Systems auf den Atmungsverlauf in verschiedenen Lungenbezirken. Pfluegers Arch Ges Physiol Menschen Tiere 1915; 162: 225-299.

17. Hantos Z, Daroczy B, Klebniczki J, Dombos K, Nagy S. Parameter estimation of transpulmonary mechanics by a non-linear inertive model. J Appl Physiol: Respirat Environ Exercise Physiol 1982; 52: 955-963. 